\title{
The pyruvate kinase activator mitapivat reduces hemolysis and improves anemia in a $\beta$-thalassemia mouse model
}

\author{
Alessandro Matte, ${ }^{1}$ Enrica Federti, ${ }^{1}$ Charles Kung, ${ }^{2}$ Penelope A. Kosinski, ${ }^{2}$ Rohini Narayanaswamy, ${ }^{2}$ Roberta Russo, ${ }^{3}$ \\ Giorgia Federico, ${ }^{3}$ Francesca Carlomagno, ${ }^{3}$ Maria Andrea Desbats, ${ }^{4}$ Leonardo Salviati, ${ }^{4}$ Christophe Leboeuf, ${ }^{5,6,7}$ \\ Maria Teresa Valenti, ${ }^{1}$ Francesco Turrini, ${ }^{8}$ Anne Janin, ${ }^{5,6,7}$ Shaoxia Yu, ${ }^{2}$ Elisabetta Beneduce, ${ }^{1}$ Sebastien Ronseaux, ${ }^{2}$ \\ Iana Iatcenko, ${ }^{1}$ Lenny Dang, ${ }^{2}$ Tomas Ganz, ${ }^{9}$ Chun-Ling Jung, ${ }^{9}$ Achille Iolascon, ${ }^{3}$ Carlo Brugnara, ${ }^{10}$ and Lucia De Franceschi \\ 'Department of Medicine, University of Verona, and Azienda Ospedaliera Universitaria Verona, Policlinico GB Rossi, Verona, Italy. ${ }^{2}$ Agios Pharmaceuticals, Inc., Cambridge, Massachusetts, USA. ${ }^{3}$ Dipartimento \\ di Medicina Molecolare e Biotecnologie Mediche, Università degli Studi di Napoli Federico Il, and CEINGE Biotecnologie Avanzate, Naples, Italy. ${ }^{4}$ Clinical Genetics Unit, Department of Women and Children's \\ Health, University of Padova, and Fondazione Istituto di Ricerca Pediatrica Città della Speranza, Padova, Italy. ${ }^{5}$ Institut National de la Santé et de la Recherche Médicale (INSERM), Paris, France. ${ }^{6}$ Université \\ Paris 7 - Denis Diderot, Paris, France. ${ }^{7}$ AP-HP, Hôpital Saint-Louis, Paris, France. ${ }^{8}$ Department of Oncology, University of Torino, Torino, Italy. ${ }^{9}$ Department of Medicine, David Geffen School of Medicine at \\ University of California Los Angeles, Los Angeles, California, USA. ${ }^{10}$ Department of Laboratory Medicine, Boston Children's Hospital, Harvard Medical School, Boston, Massachusetts, USA.
}

Anemia in $\beta$-thalassemia is related to ineffective erythropoiesis and reduced red cell survival. Excess free heme and accumulation of unpaired $\alpha$-globin chains impose substantial oxidative stress on $\beta$-thalassemic erythroblasts and erythrocytes, impacting cell metabolism. We hypothesized that increased pyruvate kinase activity induced by mitapivat (AG-348) in the $\mathrm{Hbb}^{\text {th } 3 /+}$ mouse model for $\beta$-thalassemia would reduce chronic hemolysis and ineffective erythropoiesis through stimulation of red cell glycolytic metabolism. Oral mitapivat administration ameliorated ineffective erythropoiesis and anemia in $\mathrm{Hbb}^{\text {th3/++}}$ mice. Increased ATP, reduced reactive oxygen species production, and reduced markers of mitochondrial dysfunction associated with improved mitochondrial clearance suggested enhanced metabolism following mitapivat administration in $\beta$-thalassemia. The amelioration of responsiveness to erythropoietin resulted in reduced soluble erythroferrone, increased liver Hamp expression, and diminished liver iron overload. Mitapivat reduced duodenal Dmt1 expression potentially by activating the pyruvate kinase M2-HIF2 $\alpha$ axis, representing a mechanism additional to Hamp in controlling iron absorption and preventing $\beta$-thalassemia-related liver iron overload. In ex vivo studies on erythroid precursors from patients with $\beta$-thalassemia, mitapivat enhanced erythropoiesis, promoted erythroid maturation, and decreased apoptosis. Overall, pyruvate kinase activation as a treatment modality for $\beta$-thalassemia in preclinical model systems had multiple beneficial effects in the erythropoietic compartment and beyond, providing a strong scientific basis for further clinical trials.

\section{Introduction}

Ineffective erythropoiesis and reduced red cell survival are key determinants of the anemia and other comorbidities in $\beta$-thalassemia (1-3). Reduced $\beta$-chain production leads to a decoupling from heme production, resulting in an excess of unpaired $\alpha$-globin chains and increased free heme. This process results in massive production of ROS with severe cellular oxidative damage, leading to a block in cell maturation, increased apoptosis, and ineffective erythropoiesis. In addition, increased ROS in maturing reticulocytes and erythrocytes cause hemolysis and shorten red cell survival in the peripheral circulation $(4,5)$.

Authorship note: $\mathrm{AM}, \mathrm{EF}$, and CK contributed equally to this study.

Conflict of interest: LDF received research funding from Agios. CK, PAK, RN, SY, SR and $L D$ are Agios employees and stockholders.

Copyright: ( 2021, American Society for Clinical Investigation.

Submitted: September 14, 2020; Accepted: March 31, 2021; Published: May 17, 2021

Reference information: / Clin Invest. 2021;131(10):e144206.

https://doi.org/10.1172/JCl144206.
Erythropoiesis is a metabolically demanding process: erythroid maturation strictly depends on intracellular cyclic adenosine nucleotides and ATP, and mitochondrial biogenesis plays a crucial role in ATP generation (6-9). Reduced ATP concentrations have been reported in $\beta$-thalassemic human red cells compared with healthy controls $(10,11)$. Abnormal mitochondrial clearance in maturing erythroid cells from hemoglobin $(\mathrm{Hb}) \mathrm{E} / \beta$-thalassemia patients has also been described (12).

Recently, Zhang et al. described altered gene expression of some constituents of the glycolytic pathway in murine $\beta$-thalassemic erythroid precursors, suggesting that erythrocyte metabolism and glycolysis are also affected by ineffective erythropoiesis (4). The amelioration of ineffective erythropoiesis observed in $\beta$-thalassemia mice treated with rapamycin, an mTOR inhibitor, is associated with downregulation of metabolic enzymes, including pyruvate kinase M2 (PKM2) (4). The ROS-mediated inhibition of PKM2 might redirect glucose to the pentose phosphate pathway to counteract oxidation $(13,14)$. Chronic oxidation overwhelms antioxidant cell capacity in $\beta$-thalassemic erythroid cells, as indicated by lower 
activity of reduced glutathione (GSH) and upregulation of peroxiredoxin-2, an NADPH-dependent cytoprotective system (15-17).

Pyruvate kinase plays a key role in cell metabolism, as it mediates the final step of glycolysis that yields 2 ATP and 2 pyruvate molecules per glucose molecule. It is essential for the survival of mature red blood cells and is necessary to sustain the TCA cycle in red blood cell precursors. Four different isoforms have been described: PKL, PKR (both encoded by Pklr and expressed in liver and duodenum, or erythroid cells, respectively), PKM1, and PKM2 (both present in multiple tissues, refs. 18-20). The expression of PKM2 is inducible in response to oxidation or hypoxia, whereas PKL/PKR are expressed constitutively (18-20). Patients with pyruvate kinase deficiency, an autosomal recessive disease caused by reduced PKR activity, develop chronic hemolytic anemia associated with signs of ineffective erythropoiesis. Of note, the expression of residual PKM2 has recently been reported in a patient with pyruvate kinase deficiency, supporting the importance of pyruvate kinase for erythroid cells (21). Studies in mouse models genetically lacking PKR have shown extramedullary erythropoiesis associated with ineffective erythropoiesis, further supporting the role of PKR in erythroid maturation (22-25).

Mitapivat (AG-348) is a small-molecule activator of pyruvate kinase that binds to an allosteric pocket to stabilize the active tetrameric form and enhance its affinity for its substrate, phosphoenolpyruvate (26-28). A phase 2 clinical trial of mitapivat in adult patients with pyruvate kinase deficiency who were not receiving regular transfusions demonstrated that the molecule elicited a rapid and sustained increase in $\mathrm{Hb}$ levels in approximately $50 \%$ of treated patients and was generally well tolerated, with adverse events being mainly low grade and transient (26). Interim analysis of an ongoing phase 2 study of mitapivat in patients with non-transfusion-dependent thalassemia (NCT03692052) demonstrated that $92.3 \%$ (12 of 13 evaluable patients) achieved an $\mathrm{Hb}$ increase from baseline of $\geq 1 \mathrm{~g} / \mathrm{dL}$, including 4 of 4 patients with $\alpha$-thalassemia and 8 of 9 patients with $\beta$-thalassemia (29).

We studied the effects of mitapivat in a mouse model of $\beta$-thalassemia, investigating the impact on erythropoiesis, hemolysis, anemia, iron overload, and the mechanisms involved therein. These data on the pharmacological targeting of ATP generation as a means of ameliorating $\beta$-thalassemia-associated anemia are particularly valuable for informing the development of new treatment strategies. This is especially relevant since clinical safety and tolerability for mitapivat are already available and it is currently being evaluated as a potential therapy for patients with thalassemia.

\section{Results}

Treatment with mitapivat improved anemia in a mouse model for $\beta$-thalassemia. The expression of pyruvate kinase isoforms PKR and PKM2 was evaluated in red cells from WT mice and the $\mathrm{Hbb}^{\text {th } 3 /+}$ mouse model of $\beta$-thalassemia, which mimics the severity of human $\beta$-thalassemia intermedia $(4,30)$. We analyzed red cells as a whole population or fractionated them according to cell density into 2 main fractions: fraction 1 (F1), enriched in reticulocytes; and fraction 2 (F2), containing the densest red cells and older erythrocytes (31).

The expression of both PKR and PKM2 was higher in both F1 and $\mathrm{F} 2$ red cells from $\mathrm{Hbb}^{\mathrm{th} 3 /+}$ mice compared with WT cells (Sup- plemental Figure 1A; supplemental material available online with this article; https://doi.org/10.1172/JCI144206DS1). Pyruvate kinase activity was elevated in $\mathrm{Hbb}^{\text {th } 3 /+}$ cells compared with WT cells, consistent with the observed expression levels (Supplemental Figure 1B). As expected, ROS were markedly increased in $\mathrm{Hbb}^{\text {th } 3 /+}$ erythrocytes when compared with WT erythrocytes (Supplemental Figure 1C). Notably, ROS levels were higher in $\mathrm{Hbb}^{\text {th3/+ }} \mathrm{F} 1$ than in $\mathrm{Hbb}^{\text {th } 3 /+} \mathrm{F} 2$, most likely because the more severely damaged red cells are rapidly removed from the peripheral circulation $(31,32)$.

In sorted $\mathrm{Hbb}^{\text {th } 3 /+}$ polychromatic and orthochromatic erythroblasts, the expression of PKM2 was again increased when compared with WT mice (Supplemental Figure 1D). The increased expression of PKM2 in $\mathrm{Hbb}^{\text {th } 3 /+}$ erythroid cells is of particular interest: pyruvate kinase is a key enzyme in the final step of glycolysis, and upregulation of PKM2 may be a compensatory response to oxidation as observed in other cell models (33) and in a patient with pyruvate kinase deficiency (21). The importance of PKM2 expression in response to cellular stress is also supported by evidence in hematopoietic murine cells genetically lacking PKM2 (34). In $\beta$-thalassemia, the increased expression of PKM2 might be compensatory for PKR activity to ensure cell maturation when faced with oxidative stress.

We then asked whether further pharmacological activation of pyruvate kinase may be of benefit in $\beta$-thalassemia. The pyruvate kinase activator, mitapivat, was either administered by gavage (50 mg/kg twice a day) for 21 days or added to the mouse diet for 56 days to minimize the possible stress related to animal manipulation. As shown in Figure 1A, mitapivat treatment resulted in markedly improved red cell morphology compared with baseline in $\mathrm{Hbb}^{\text {th } 3 /+}$ mice. $\mathrm{Hbb}^{\text {th } 3 /+}$ smears showed typical hypochromic red cells, marked polychromasia, and various forms of fragmented erythrocytes (poikilocytes) associated with stressed erythropoiesis and hemolysis. Poikilocytosis improved visibly after 14 and 21 days of treatment with mitapivat. $\mathrm{Hb}$, mean corpuscular volume (MCV), and mean corpuscular $\mathrm{Hb}(\mathrm{MCH})$ also increased in $\mathrm{Hbb}^{\text {th } 3 /+}$ mice after 3 weeks of mitapivat treatment (Figure 1B and Supplemental Table 1). A significant decrease in absolute reticulocyte counts was also found in mitapivat-treated $\mathrm{Hbb}^{\mathrm{th} 3 /+}$ mice $(P<0.05)$, in conjunction with increased reticulocyte MCV (Figure $1 \mathrm{C}$ and Supplemental Table 1). Similar results were also observed in $\mathrm{Hbb}^{\text {th } 3 /+}$ mice treated with mitapivat for 56 days (Supplemental Figure 2, A and B). Consistent with these data, we also found a marked reduction in circulating erythroblasts (Supplemental Figure 2C).

The amelioration of anemia and red cell indices was associated with a significant reduction in $\alpha$-globin membrane precipitates $(P<0.05)$ and an increase in the fraction of soluble $\mathrm{Hb}$ in red cells from mitapivat-treated $\mathrm{Hbb}^{\text {th3/++}}$ mice compared with vehicle-treated animals (Figure 1D). A significant decrease of ROS in circulating $\mathrm{Hbb}^{\text {th } 3 /+}$ erythrocytes was observed after 3 weeks of mitapivat treatment $(P<0.05$; Figure $1 \mathrm{E})$, which was associated with an increased GSH/glutathione disulfide (GSSG) ratio (Figure $1 F$ ). Hemichromes bound to the membrane of red cells from mitapivat-treated $\mathrm{Hbb}^{\text {th } 3 /+}$ mice were also significantly reduced compared with vehicle-treated animals $(P<0.05$; Supplemental Figure 2D). There was a trend toward increased ATP levels in blood from treated animals (Supplemental Figure 2E), consistent with activation of pyruvate kinase. Plasma total bilirubin, 
A

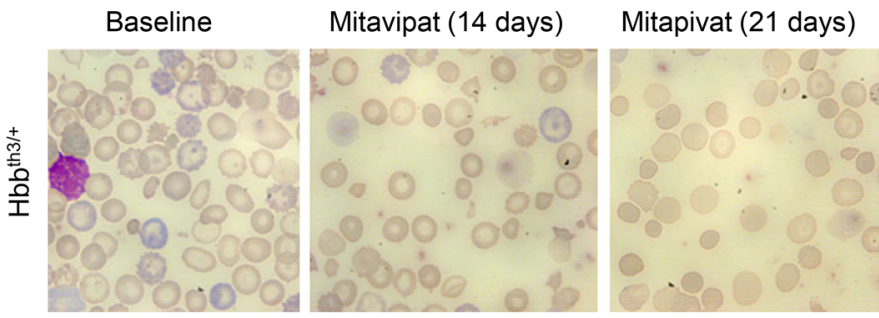

B
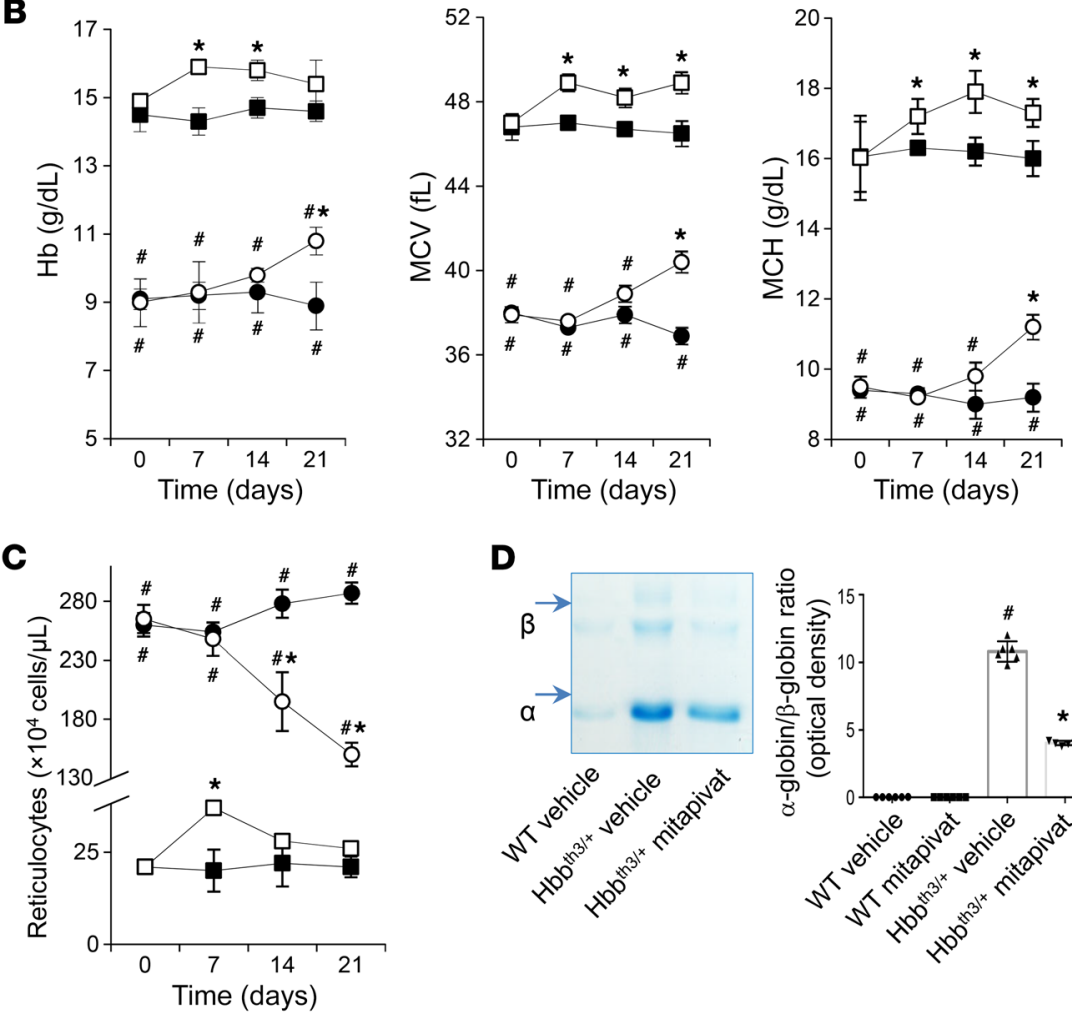

D
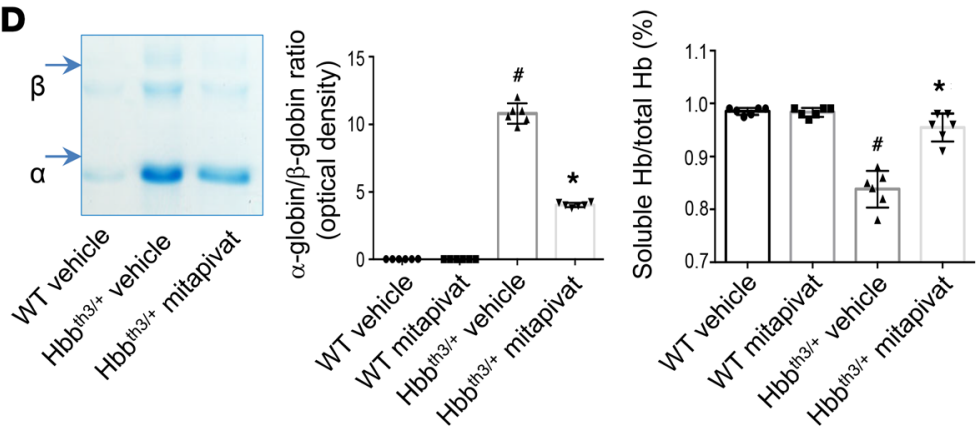

E

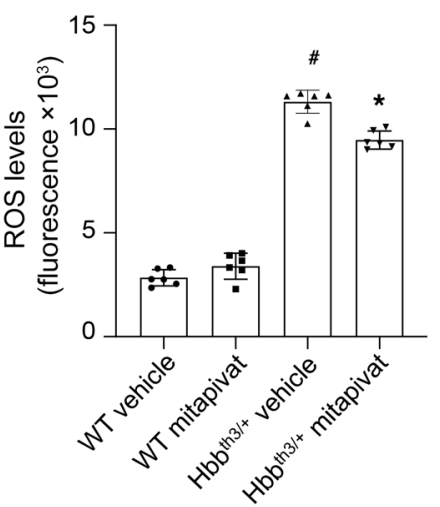

$\mathbf{F}$

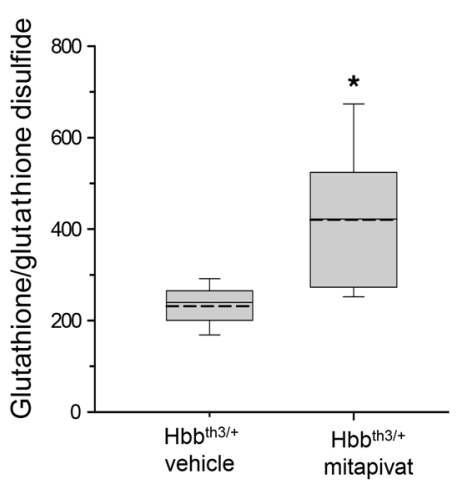

- WT vehicle

$\rightarrow$ WT mitapivat

$\longrightarrow \mathrm{Hbb}^{\text {th } 3 /+}$ vehicle

- $\mathrm{Hbb}^{\mathrm{th} 3 /+}$ mitapivat

(n)<smiles>C1CC1</smiles>

G

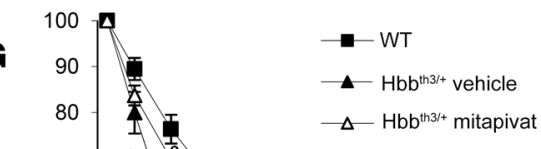

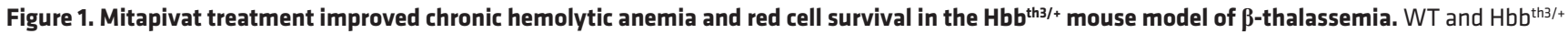
mice were treated with vehicle or mitapivat $50 \mathrm{mg} / \mathrm{kg}$ twice daily. (A) Erythrocyte morphology. One representative image from 8 with similar results shown. Original magnification $\times 100$. (B and $\mathbf{C}$ ) $\mathrm{Hb}, \mathrm{MCV}, \mathrm{MCH}$, and reticulocyte count in WT and Hbb ${ }^{\text {th } 3 /+}$ mice treated with vehicle or mitapivat. Data are mean \pm SD $(n=6)$. ( $\mathbf{D}$, left panel) Triton acid-urea gel electrophoresis of red cell membrane from WT and Hbb ${ }^{\text {th } 3 /+}$ mice treated with vehicle or mitapivat (21 days). Arrows show $\alpha$-globin and $\beta$-globin associated with red cell membrane. (D, middle panel) Gel quantification expressed as $\alpha$-globin/ $\beta$-globin ratio to Hb. Data are mean \pm SD $(n=6)$. (D, right panel) Total and soluble Hb (Drabkin's method) in hemolysates from Hbb ${ }^{\text {th } 3 /+}$ mice treated with vehicle or mitapivat ( 21 days). Data are mean \pm SD $(n=6)$. (E) ROS levels in red cells from WT and Hbb ${ }^{\text {th } 3 /+}$ mice treated with vehicle or mitapivat. Data are mean \pm SD ( $n=6$ each). (F) Difference in the glutathione/glutathione disulfide ratio between $\mathrm{Hbb}^{\text {th } 3 /+}$ mice treated with vehicle or mitapivat ( $n=5-9$ for each). Boxes represent 25 th to 75 th percentiles; continuous and dashed lines mark median and mean values, respectively. Whiskers indicate 90 th and 10 th percentiles. ${ }^{*} P<0.05$ by 2 -tailed $t$ test. (G) Survival of CFSE-labeled red cells from WT $(n=4)$ and Hbb ${ }^{\text {th } 3 /+}$ mice treated with vehicle or mitapivat $(n=3$ each group). Data are mean \pm SD. (B, C, and $\mathbf{G}){ }^{*} P<0.05$ compared with WT and ${ }^{*} P<0.05$ compared with vehicle by 1 -way ANOVA with Dunnett's longitudinal comparison test. ( $\mathbf{D}$ and $\mathbf{E}$ ) ${ }^{*} P<0.02$ compared with WT and ${ }^{*} P<0.05$ compared with vehicle-treated mice. 
indirect bilirubin, and lactate dehydrogenase, known markers of hemolysis, were reduced following 21 days of mitapivat treatment (Supplemental Figure 3, A and B). The survival of red cells from mitapivat-treated $\mathrm{Hbb}^{\text {th } 3 /+}$ mice was significantly improved, with an estimated half-life of $14 \pm 0.4$ days, compared with $9.6 \pm 0.9$ days in vehicle-treated $\mathrm{Hbb}^{\text {th } 3 /+}$ animals $(P<0.05)$ and $18.9 \pm 0.5$ days in WT controls (Figure 1G and Supplemental Table 2). No change in either creatinine or blood urea nitrogen was observed in mitapivat-treated $\mathrm{Hbb}^{\text {th } 3 /+}$ mice when compared with vehicle-treated animals (Supplemental Figure 3C).

WT mice treated with mitapivat showed a slight but significant increase in $\mathrm{Hb}(P<0.05)$ as well as an increase in $\mathrm{MCV}, \mathrm{MCH}$, and reticulocyte counts at 7 days of treatment, which generally returned toward baseline values at 14 and 21 days of treatment (Figure 1, B and C). These data suggest either a transient erythroid stimulation by mitapivat at day 7 of treatment, returning to baseline at day 21 in mitapivat-treated WT animals, or an increase in efficiency of erythropoiesis during development of late-stage precursors into reticulocytes.

Together, our findings in $\mathrm{Hbb}^{\text {th } 3 /+}$ mice suggest that oral administration of mitapivat reduces hemolysis, improves red cell survival, and increases Hb levels.

A reduction in plasma erythropoietin (EPO) was observed in $\mathrm{Hbb}^{\text {th } 3 /+}$ mice treated with mitapivat (Figure $2 \mathrm{~A}$ ). $\beta$-thalassemia is characterized by ineffective erythropoiesis with impaired erythroid maturation, leading to an expansion of immature erythroblast populations. In $\mathrm{Hbb}^{\text {th } 3 /+}$ mice, mitapivat markedly decreased extramedullary erythropoiesis as supported by a reduction in the spleen/body weight ratio, decreased spleen iron content, and a reduction in spleen erythropoietic activity (Figure 2, B and C and Supplemental Figure 4A). Moreover, 3 weeks of mitapivat treatment in $\mathrm{Hbb}^{\text {th } 3 /+}$ mice resulted in an improvement of bone marrow erythroblast maturation index, assessed as the ratio between early (pro-plus basophilic) and late (polychromatic plus orthochromatic) erythroblasts (Figure 2D). This was associated with upregulation of Cish and Serpin-3, genes strictly dependent on Stat5 function, supporting an increase in plasma EPO responsiveness in mitapivat-treated $\mathrm{Hbb}^{\text {th } 3 /+}$ mice (Supplemental Figure 4B).

A marked increase in pyruvate kinase activity was observed in polychromatic and orthochromatic erythroblasts from mitapivat-treated $\mathrm{Hbb}^{\text {th } 3 /+}$ mice (Supplemental Figure $4 \mathrm{C}$ ). This was associated with a significant increase in ATP in sorted erythroid cells from spleen of mitapivat-treated $\mathrm{Hbb}^{\text {th } 3 /+}$ mice when compared with vehicle-treated animals $(P<0.05$; Figure $2 \mathrm{E})$. Mitapivat administration also resulted in a marked decrease in ROS in maturing $\mathrm{Hbb}^{\text {th } 3 /+}$ erythroblasts (Figure 2F). Apoptotic (annexin $\left.-\mathrm{V}^{+}\right)$erythroblasts were significantly reduced in both spleen and bone marrow of mitapivat-treated $\mathrm{Hbb}^{\text {th } 3 /+}$ mice $(P<0.05$ for both; Figure $2 \mathrm{G}$ ). The mitapivat-induced amelioration of ineffective erythropoiesis was accompanied by decreased Erfe (an erythroid regulator of hepcidin) expression in sorted erythroblasts as well as reduced erythroferrone (ERFE) plasma values (Figure 2, $\mathrm{H}$ and I). These data suggest that pyruvate kinase activation by mitapivat ameliorates the ineffective erythropoiesis observed in $\mathrm{Hbb}^{\mathrm{th} 3 /+}$, improving EPO responsiveness and reducing oxidative stress.

Mitapivat improved mitochondrial dysfunction in $\mathrm{Hbb}^{\text {th } 3 /+}$ mouse erythroblasts. Previous studies have suggested that mitochondri- al dysfunction is an important feature of ineffective erythropoiesis in $\beta$-thalassemia $(12,35)$. Using MitoTracker, we observed that $\mathrm{Hbb}^{\text {th } 3 /+}$ erythroblasts from mitapivat-treated mice exhibited increased clearance of mitochondria during maturation compared with vehicle-treated animals (Figure 3A). We then evaluated mRNA expression of genes involved in mitochondrial biogenesis and in quality control of inner mitochondrial membrane proteins, including (a) Atp6, Mtco1, and Cytb; (b) Pgc1a, a master regulator of mitochondrial biogenesis; and (c) Yme1l, an oxidation-sensitive protease with mitochondrial-specific chaperone-like function, which provides a mechanism to sequester damaged mitochondria away from a healthy mitochondrial pool $(36,37)$.

In erythroblasts from mitapivat-treated $\mathrm{Hbb}^{\text {th } 3 /+}$ mice, we observed upregulation of Atp6, Mtco1, Cytb, and Pgcla genes (Figure 3B). Although increased expression of Atp6, Mtco1, and Cytb might result from a reduction in cell oxidation, the upregulation of Pgc1a indicates a specific improvement of mitochondrial function with mitapivat treatment. This was accompanied by the downregulation of Yme1l, a mitochondria-specific chaperone, suggesting a beneficial effect of mitapivat on mitochondrial biogenesis (Figure 3C). In erythroblasts from WT mice, we observed upregulation of Atp6, Mtco1, and Cytb genes, whereas no major change was observed in Pgc1a gene expression (Supplemental Figure 4D). Data from $\mathrm{Hbb}^{\text {th3/+ }}$ mice indicate that mitapivat treatment ameliorates mitochondrial biogenesis and improves erythropoietic mitochondrial dynamics in $\beta$-thalassemia.

Mitapivat reduced liver iron overload and oxidative stress, and increased liver hepcidin expression in $\mathrm{Hbb}^{\text {th } 3 /+}$ mice. Liver iron overload is a common and severe pathological feature of $\beta$-thalassemia, a consequence of ineffective erythropoiesis and inappropriate hepcidin (Hamp) downregulation (38-40). In $\mathrm{Hbb}^{\text {th } 3 /+}$ mice, mitapivat significantly reduced liver iron overload in both hepatocytes and Kupffer cells compared with vehicle-treated animals $(P<0.05$ for both; Figure 4A). Consistently, there was a significant reduction in liver non-heme iron content in mitapivat-treated $\mathrm{Hbb}^{\text {th } 3 /+}$ mice compared with vehicle treated $\mathrm{Hbb}^{\text {th } 3 /+}$ mice $(P<0.05$; Supplemental Figure 5A). A significant reduction in liver protein oxidation, presumably linked to a reduction in iron content, was also observed $(P<0.05$; Figure 4B). As expected, on the basis of erythropoiesis improvement, mitapivat treatment resulted in upregulation of liver Hamp expression in $\mathrm{Hbb}^{\text {th } 3 /+}$ mice (Figure $4 \mathrm{C}$ ). This was associated with upregulation of $I d 1$, which is linked to the activation of the SMAD system (Figure 4D). In mitapivat-treated $\mathrm{Hbb}^{\mathrm{th} 3 /+}$ mice, we observed decreased activation of NRF2, STAT3, and NF-кB p65 (Figure 4E), with no major change in ERK1/2 activation (Supplemental Figure 5B). These data indicate a beneficial effect of mitapivat on Hamp expression, most likely via feedback registering mitapivat-induced improvements in erythropoiesis.

Mitapivat treatment reduced iron overload and Dmt1 expression in duodenums of $\mathrm{Hbb}^{\text {th } 3 /+}$ mice. Previous studies have shown that hepcidin targets ferroportin (FPN1) in the duodenum, promoting its internalization and degradation, thus modulating iron absorption (17, 41-43). Consistent with this, we observed a decrease in FPN1 staining in the duodenums of mitapivat-treated $\mathrm{Hbb}^{\mathrm{th} 3 /+}$ mice, with only some basolateral membrane localization left (Supplemental Figure 6A). Besides FPN1 downregulation, we observed a significant reduction of iron overload in duodenal enterocytes 

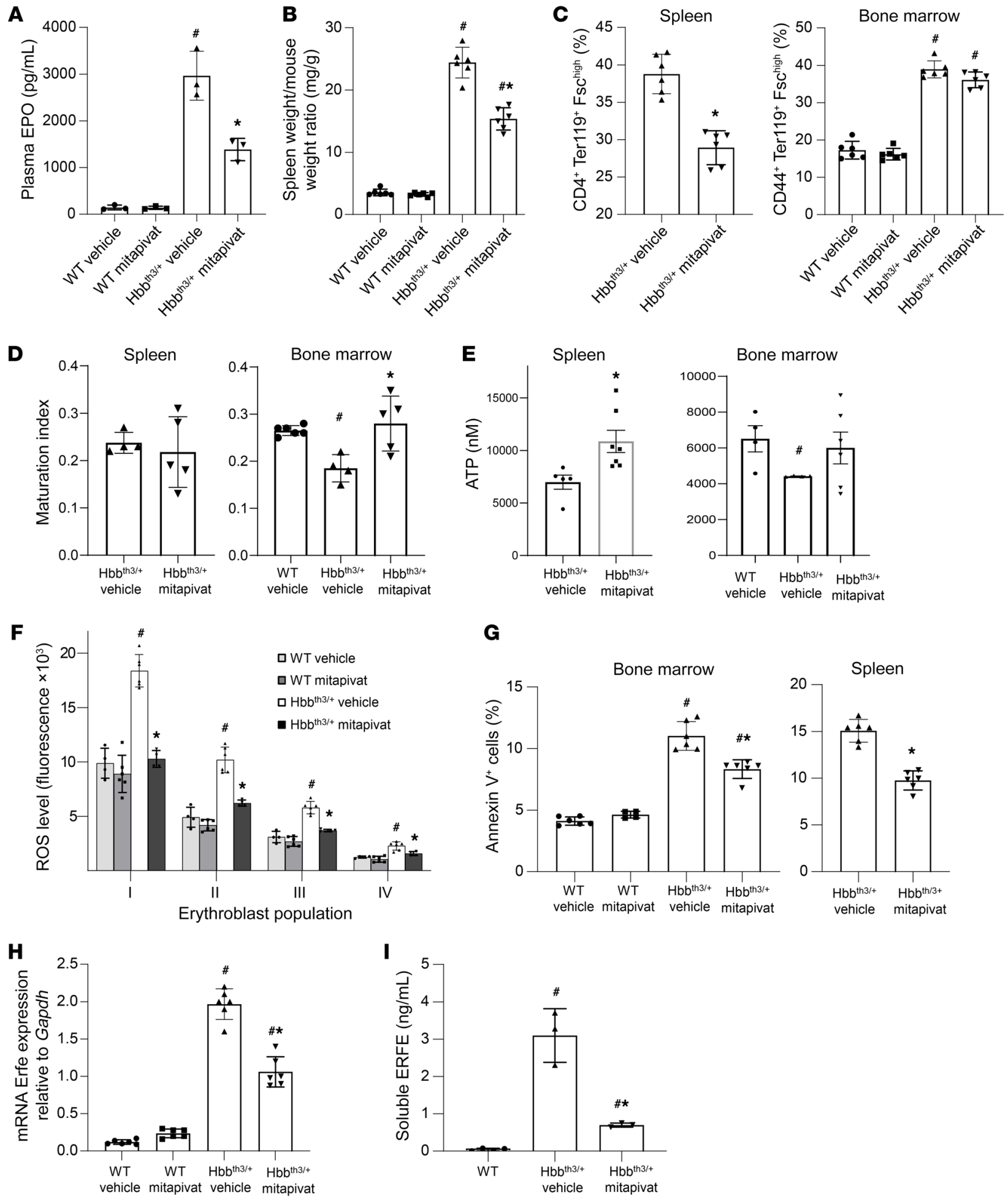

Figure 2. Mitapivat ameliorated ineffective erythropoiesis in $\mathbf{H b b}^{\text {th } 3 /+}$ mice. (A) Plasma EPO in WT and Hbb ${ }^{\text {th } 3 /+}$ mice. Data are mean \pm SD $(n=3)$. (B) Spleen weight/mouse weight ratio in WT and $\mathrm{Hbb}^{\text {th3/+ }}$ mice. Mean \pm SD $(n=6)$. (C) Flow cytometry (CD44-Ter119 and cell size markers) of bone marrow and spleen from WT and Hbb ${ }^{\text {th } 3 /+}$ mice. Mean \pm SD $(n=6)$. (D) Ratio of proerythroblasts plus basophilic erythroblasts/polychromatic plus orthochromatic erythroblasts (maturation index) in spleen and bone marrow from Hbb ${ }^{\text {th } 3 /+}$ mice by flow cytometry. Mean $\pm \mathrm{SD}\left(\mathrm{Hbb} \mathrm{b}^{\text {th } 3 /+}, n=4\right.$ vehicle-treated, $n=5$ mitapivat-treated; WT, $n=6$ ). (E) ATP content in sorted CD44-Ter119 total erythroblasts from spleen and bone marrow of $\mathrm{Hbb}^{\text {th } 3 /+}$ mice and bone marrow of WT mice. Mean \pm SEM (spleen, vehicle-treated, $n=5$, versus mitapivat-treated, $n=7 ; P<0.05$; bone marrow, WT vehicle, $n=4$, Hbb ${ }^{\text {th } 3 /+}$ vehicle-treated, $n=4$, versus mitapivat-treated, $n=6 ; P=\mathrm{NS}$ ). (F) ROS levels in erythroblasts from WT or Hbb ${ }^{\text {th } 3 /+}$ mice. Mean $\pm \mathrm{SD}$ (WT, $n=4$ vehicle-treated and $n=6$ mitapivat-treated; $\mathrm{Hbb}^{\text {th } 3 /+}, n=6$ vehicle and $n=4$ mitapivat-treated). (C) Annexin- $\mathrm{V}^{+}$erythroid cells from WT or Hbb ${ }^{\text {th } 3 /+}$ mice. Mean $\pm \mathrm{SD}$ (WT, vehicle-treated $n=6$ and mitapivat-treated $n=4 ; \mathrm{Hbb}^{\text {th } 3 /+}, n=6$ each group). (H) mRNA expression of Erfe by qRT-PCR of sorted erythroblasts from bone marrow of WT and $\mathrm{Hbb}^{\text {th } 3 /+}$ mice. Mean \pm SD, $n=6$. (I) Soluble plasma ERFE levels in WT and Hbb ${ }^{\text {th } 3 /+}$. Mean \pm SD (WT, vehicle-treated $n=4 ; \mathrm{Hbb}^{\text {th } 3 /+}, n=3$ each group). $(\mathbf{A}-\mathbf{D}, \mathbf{F}-\mathbf{I}){ }^{\#} P<0.05$ versus WT mice and ${ }^{*} P<0.05$ versus vehicle-treated mice by 2 -way ANOVA with Bonferroni multiple comparison correction. 
A

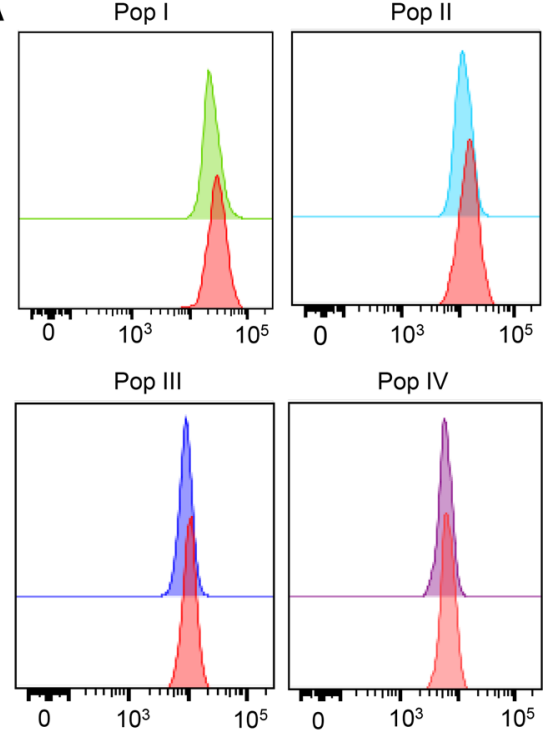

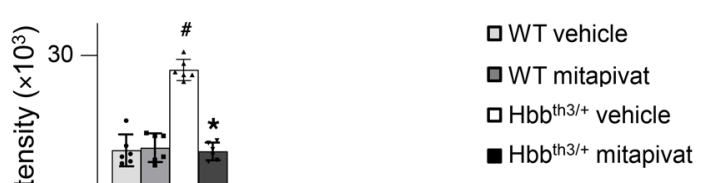

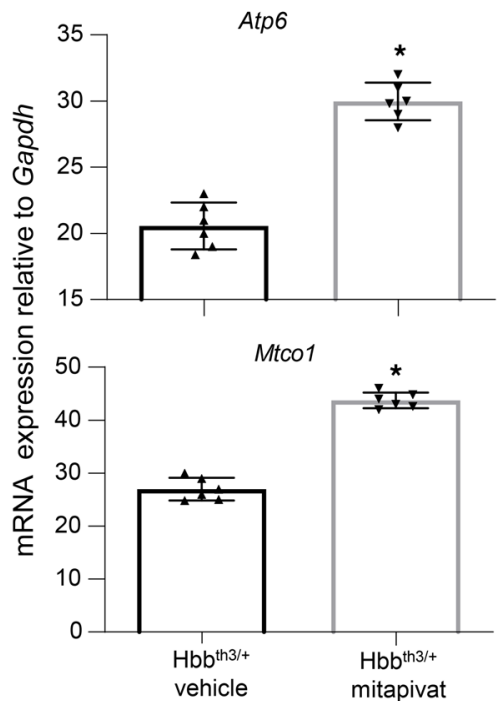

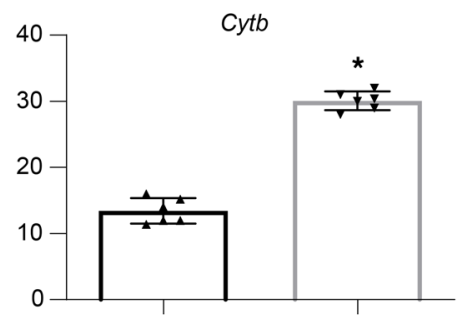

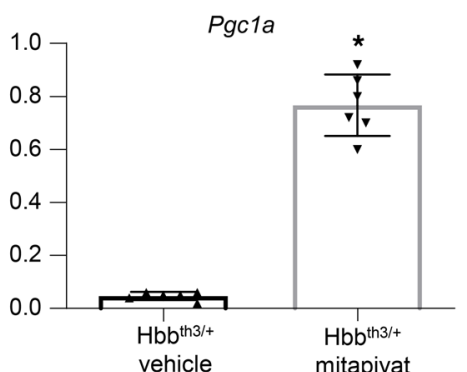

C

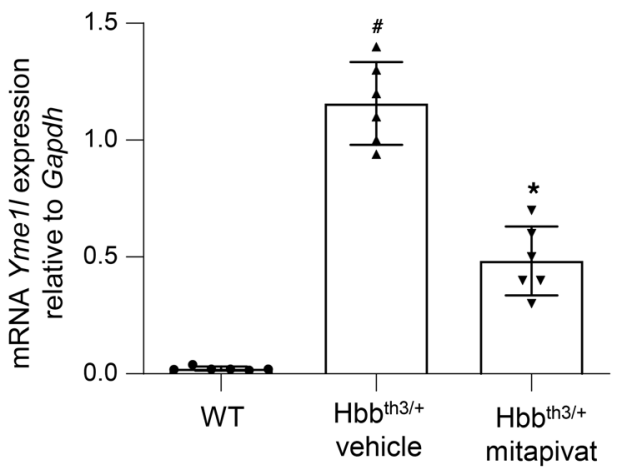

Figure 3. Mitapivat improved mitochondrial dysfunction and biogenesis in $\mathbf{H b}^{\text {th }}{ }^{\text {3/+ }}$ mice. (A, left panel) Flow cytometric analysis using MitoTracker in erythroblasts from WT (red) and Hbb ${ }^{\text {th3/+ }}$ (proerythroblasts [pop I, green], basophilic erythroblasts [pop II, blue], polychromatic erythroblasts [pop III, purple], and orthochromatic erythroblasts [pop IV, pink]) mice. One representative scatter from 4 with similar results is presented. (A, right panel) Mitochondrial content of erythroblasts from WT and $\mathrm{Hbb}^{\text {th } 3 /+}$ mice treated with vehicle or mitapivat. Data are mean \pm SD $(n=6)$. (B) mRNA expression of Atp6, Mtco1, Cytb, and Pgc1a genes by qRT-PCR on sorted erythroblasts from bone marrow of $\mathrm{Hbb}^{\text {th3/+ }}$ mice treated with vehicle or mitapivat. Data are mean \pm $\mathrm{SD}\left(n=6\right.$ per group). (C) mRNA expression of Yme1l by qRT-PCR on sorted erythroblasts from bone marrow of WT and Hbb ${ }^{\text {th } 3 /+}$ mice treated with vehicle or mitapivat. Data are mean \pm SD ( $n=6$ per group). (A-C) ${ }^{*} P<0.05$ compared with WT mice and ${ }^{*} P<0.05$ compared with vehicle-treated mice by 2 -way ANOVA with Bonferroni multiple comparison correction.

compared with age-matched, vehicle-treated $\mathrm{Hbb}^{\mathrm{th} 3 /+}$ mice $(P<$ 0.05). No overload was present in duodenums of WT mice (Figure $5 \mathrm{~A}$ ). We hypothesized that mitapivat, by increasing pyruvate kinase activity, might improve the duodenal oxidative/hypoxic intracellular environment and consequently contribute to a reduction of iron uptake by downregulation of Dmt1 expression. Indeed, mitapivat treatment also resulted in increased expression of PKR and reduced expression of stress-associated PKM2 in duodenums from $\mathrm{Hbb}^{\mathrm{th} 3 /+}$ mice compared with vehicle-treated animals, suggesting a possible contribution of pyruvate kinase to small intestine metabolic function (Figure 5B, see also Supplemental Figure
6C and ref. 20). Notably, duodenum PKM2 expression was significantly higher in vehicle-treated $\mathrm{Hbb}^{\mathrm{th} 3 /+}$ mice than in WT animals $(P<0.05)$, further suggesting that stress/hypoxic-response pathways are activated in duodenal cells of animals with $\beta$-thalassemia (Figure 5B and Supplemental Figure 6B). In addition, PKM2 was present as dimer in duodenum from $\mathrm{Hbb}^{\mathrm{th} / /}$ mice, supporting the activation of the PKM2-HIF axis (44).

As expected, in enterocytes from mitapivat-treated $\mathrm{Hbb}^{\mathrm{th} 3 /+}$ mice, we observed a reduction of HIF $2 \alpha$ and of the NF-кB p65 active form (Figure 5C) as well as of both Dmt1-iron response element (IRE) and Dmt1-non-IRE mRNA transcripts (Figure 5D and 
A
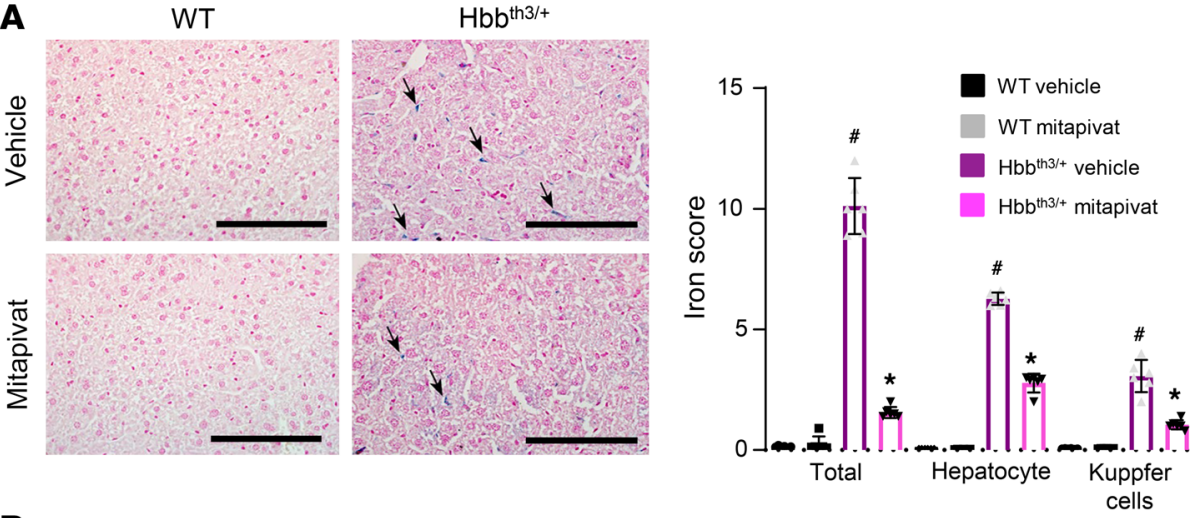

B
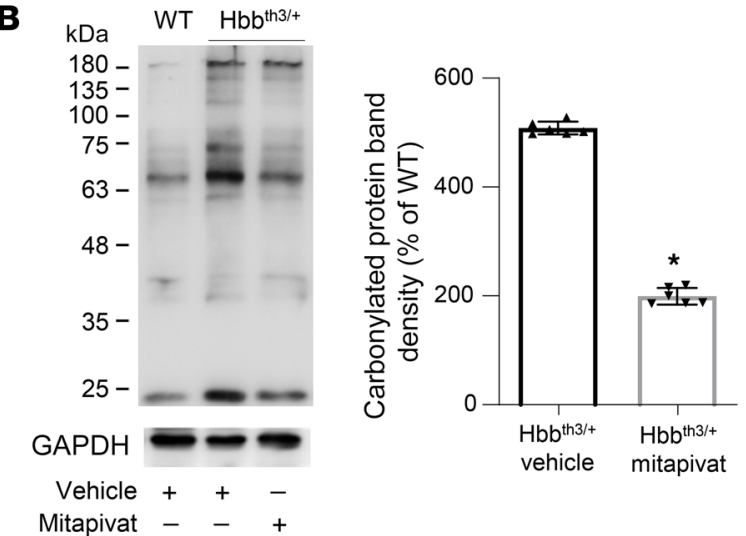

C

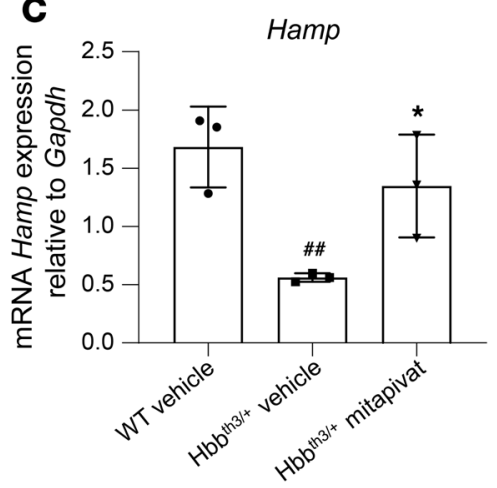

D

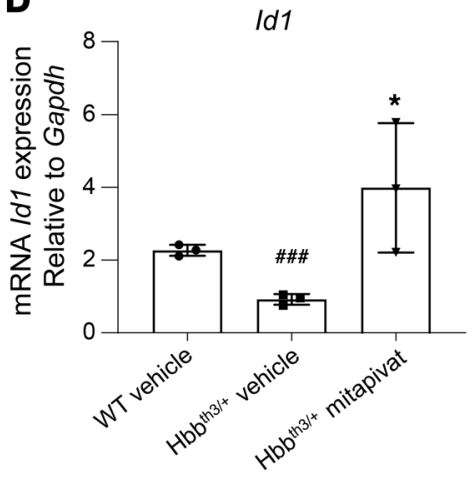

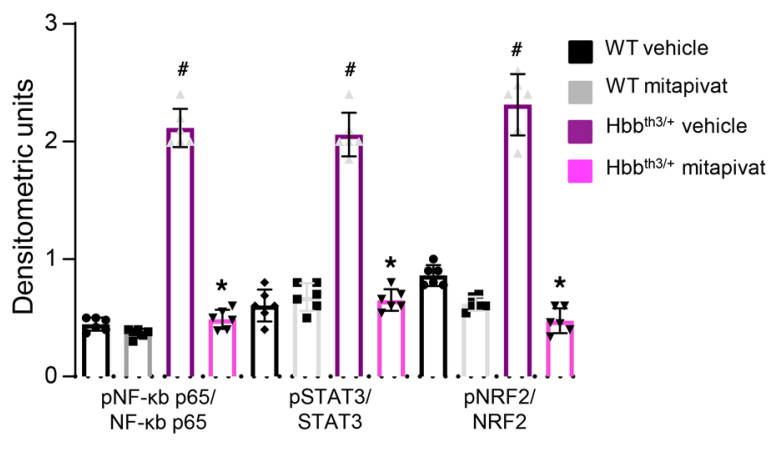

Figure 4. In $\mathrm{Hbb}^{\text {th } 3 /+}$ mice, mitapivat reduced liver iron overload and oxidative stress and increased hepcidin. (A, left panel) Iron staining (Perl's Prussian blue) in liver from WT and $\mathrm{Hbb}^{\text {th } 3 /+}$ mice treated with vehicle or mitapivat. Original magnification $\times 200$. Scale bars: $100 \mu \mathrm{M}$. One representative image from 4 with similar results. Black arrows indicate liver iron deposits. (A, right panel) Quantification of iron staining in liver. Data are mean \pm SD $(n=6) . ~ " P<0.05$ compared with WT mice and ${ }^{*} P<0.05$ compared with vehicle-treated mice by 2-way ANOVA with Bonferroni multiple comparison correction. (B) Soluble fractions of liver from $\mathrm{WT}$ and $\mathrm{Hbb}^{\mathrm{th} 3 /+}$ mice with and without mitapivat were analyzed with $12 \%$ SDSPAGE and OxyBlot. Quantification of band area was performed by densitometry and expressed as a percentage of WT. Data are mean $\pm S D, n=6$. (C and D) mRNA expression of hepcidin (Hamp) and Id1 by qRT-PCR on liver from WT and $\mathrm{Hbb}^{\text {th } 3 /+}$ mice treated with vehicle or mitapivat. Experiments were performed in triplicate. Data are mean \pm SD. ${ }^{~} P<0.05$ compared with WT mice and ${ }^{*} P<0.05$ compared with vehicle-treated mice by multiple comparisons using ANOVA; internal comparisons were calculated by unpaired 2-tailed Student $t$ test. ${ }^{\# \#} P<0.05$ compared with WT mice, ${ }^{\# \# P} P<0.02$ compared with WT mice, ${ }^{*} P<0.05$ compared with $\mathrm{HBB}^{\text {th } 3 /+}$ mice. A 2 -sided $P<0.05$ was considered statistically significant. (E, left panel) Western blot analysis with specific antibodies against phospho (p) NF- $\kappa B$ p65, NF- $K B$ p65, pSTAT3, STAT3, pNRF2, and NRF2 of liver from WT and $\mathrm{Hbb}^{\text {th } 3 /+}$ mice with vehicle or mitapivat treatment, GAPDH as loading control. One representative gel from 6 with similar results. (E, right panel) Densitometric analyses of the Western blots. Data are mean $\pm S D(n=6)$. (B and E) ${ }^{\#} P<0.05$ compared with WT mice and ${ }^{*} P$ $<0.05$ compared with vehicle-treated mice by 2 -way ANOVA with Bonferroni multiple comparison correction.

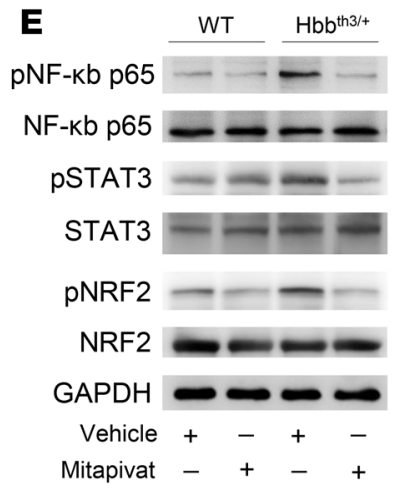

Supplemental Figure 7). In agreement, we found up- and downregulation of PKM2-HIF targets beside Dmt1 such as Ldha, Pdk1, and Slc2a1 expression (Supplemental Figure 7) (45).

Our data suggest a novel contribution of PKM2 to iron homeostasis of $\mathrm{Hbb}^{\mathrm{th} 3 /+}$ mice. Mitapivat promotes the activation of pyruvate kinase and downregulation of the stress responseassociated expression of PKM2. This in turn reduces activation of redox/hypoxia-related transcription factors such as the NF-kB/ HIF system with downregulation of Dmt1 gene expression, which directly impacts iron absorption, resulting in a substantial clear- 
A

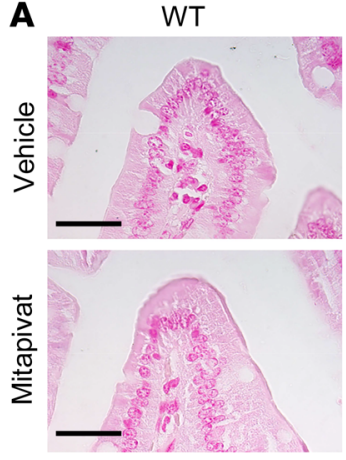

B

C

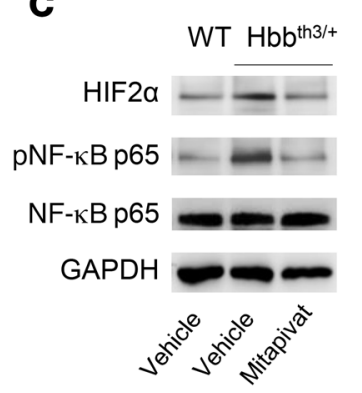

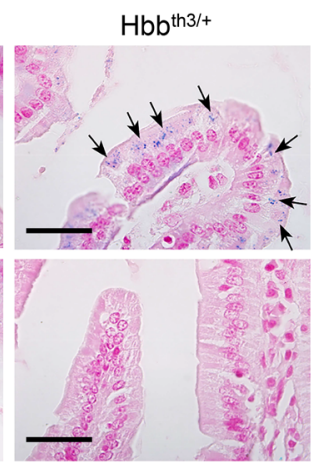

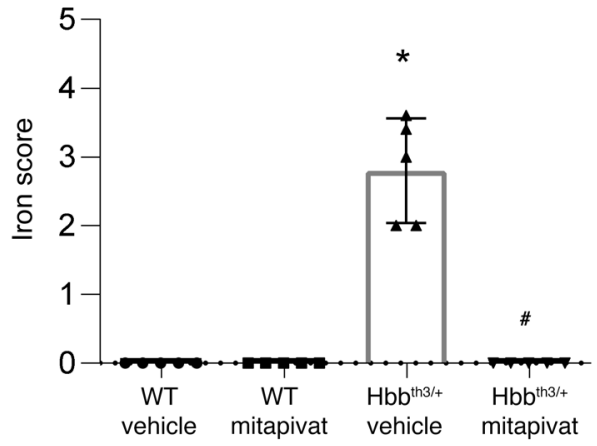

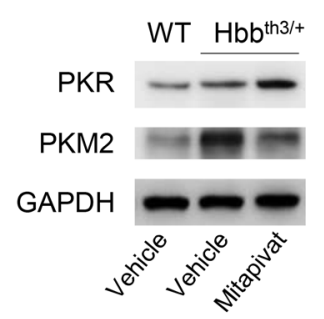

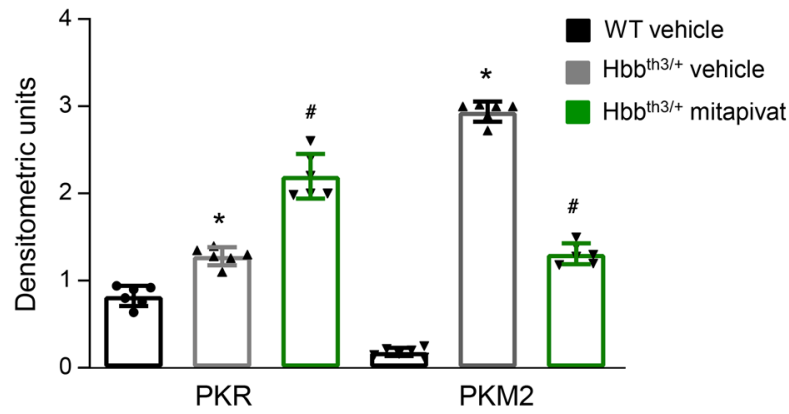

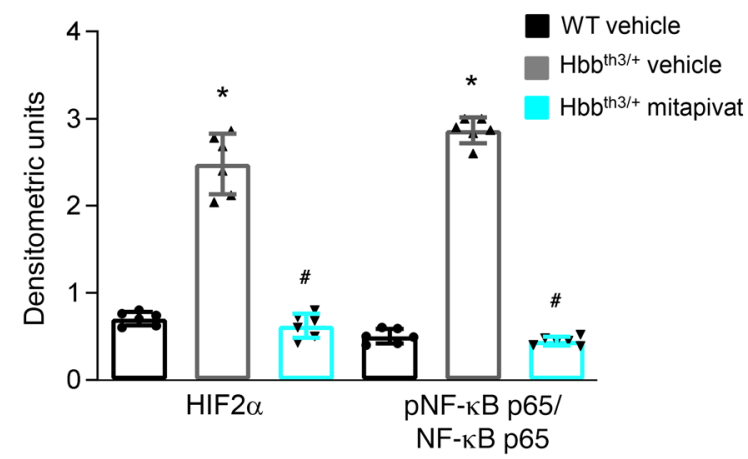

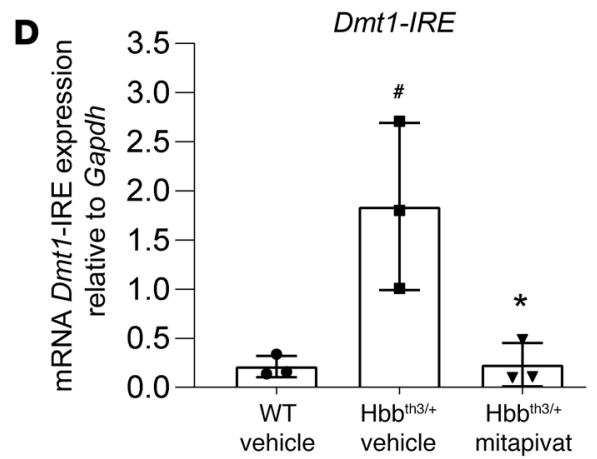

Figure 5. Mitapivat-treated $\mathbf{H b b}^{\text {th } 3 /+}$ mice showed reduced ferroportin, reduced duodenum iron overload, and downregulation of the HIF2 $\alpha-D m t 1$ signaling axis. (A) Iron staining (Perl's Prussian blue) in duodenums from WT and $\mathrm{Hbb}^{\text {th } 3 /+}$ mice treated with vehicle or mitapivat. Original magnification $\times 400$. Scale bars: $100 \mu \mathrm{M}$. One representative image from 4 with similar results. Arrows indicate iron deposits. (A, right panel) Quantification of iron staining. Data are mean $\pm \mathrm{SD}(n=5) .{ }^{*} P<0.05$ compared with WT or ${ }^{\#} P<0.05$ compared with vehicle-treated $\mathrm{Hbb}^{\text {th } 3 /+}$ mice by 2 -tailed $t$ test. (B, upper panel) Western blot analysis using specific antibodies against PKR and PKM2 of duodenums from WT and Hbb ${ }^{\text {th } 3 /+}$ mice treated with vehicle or mitapivat, GAPDH as loading control. One representative gel from 4 with similar results. (B, lower panel). Densitometric analyses of the Western blots, mean \pm SD $(n=6) .{ }^{*} P<0.05$ compared with WT by 1-way ANOVA with Bonferroni multiple comparison correction. ${ }^{*} P<0.05$ compared with vehicle-treated Hbb ${ }^{\text {th }} /++$ mice by 1-way ANOVA with Bonferroni multiple comparison correction. (C, left panel) Western blot analysis with specific antibodies against HIF2 $\alpha$, pNF- $\mathrm{kB}$ p65, and NF- $\mathrm{kB}$ p65 of duodenums from WT and $\mathrm{Hbb}^{\mathrm{th} /+}$ mice treated with vehicle or mitapivat, GAPDH as loading control. One representative gel from 4 with similar results. (C, right panel) Densitometric analyses of the Western blots, mean \pm SD $(n=6)$. ${ }^{*} P<0.05$ compared with WT or ${ }^{\#} P<0.05$ compared with vehicle-treated $\mathrm{Hbb}^{\text {th3/++ }}$ mice by 1-way ANOVA with Bonferroni multiple comparison correction. (D) mRNA expression of Dmt1-IRE by qRT-PCR on duodenums from WT and $\mathrm{Hbb}^{\text {th } 3 /+}$ mice treated with vehicle or mitapivat. Experiments were performed in triplicate. Data are mean $\pm \mathrm{SD}$. ${ }^{\#}<<0.02$ compared with WT mice and ${ }^{*} P<0.05$ compared with vehicle-treated mice by 2-way ANOVA with Tukey's multiple comparisons test.

ance of iron overload from duodenal enterocytes and possibly from the entire organism.

Mitapivat enhanced in vitro human erythropoiesis. We evaluated the effect of mitapivat on human erythropoiesis in an in vitro culture system based on $\mathrm{CD} 34^{+}$cells derived from peripheral blood of healthy controls and patients with $\beta$-thalassemia intermedia (46-48). This culture system recapitulates the ineffective erythropoiesis observed in vivo in $\beta$-thalassemia, exhibiting increased apoptosis and disturbed maturation.
In this in vitro system, mitapivat induced a slight increase in cell numbers in the late phase of erythropoiesis compared with vehicle-treated cells (Supplemental Figure 8A). This was associated with a substantial increase of orthochromatic erythroblasts at 11 and 14 days of cell culture compared with vehicle-treated $\beta$-thalassemic cells (Supplemental Figure 8, B and C). Improved $\beta$-thalassemia erythroid maturation was also supported by a significant decrease in annexin $\mathrm{V}^{+}$cells at 11 (pro- and intermediate erythroblasts) and 14 days of cell culture (pro-, intermediate, and late erythroblasts) 


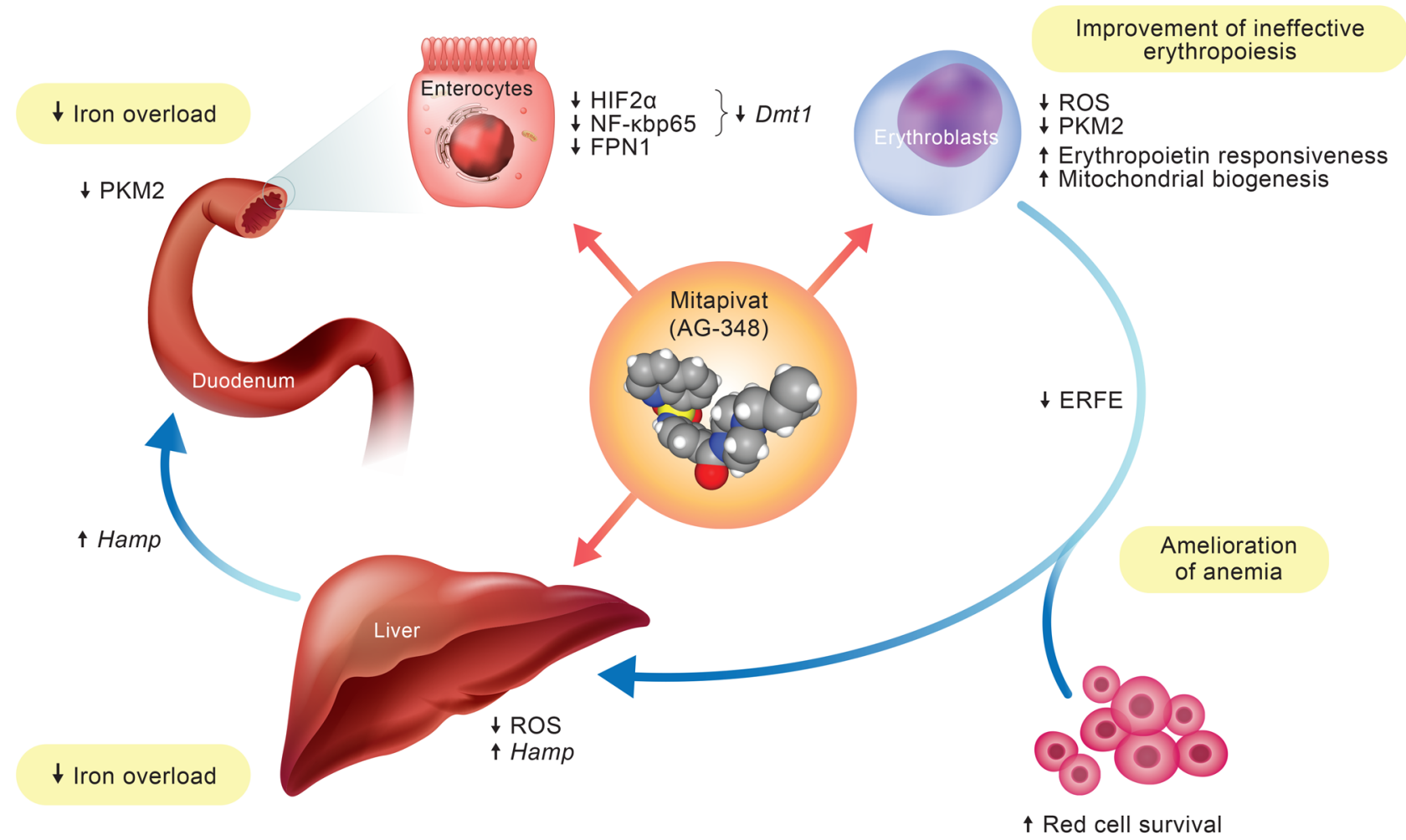

Figure 6. Schematic diagram of mitapivat actions in $\boldsymbol{\beta}$-thalassemic mouse model. The downstream effects of mitapivat on erythropoiesis and iron homeostasis. Mitapivat improves anemia, hemolysis, and red cell survival, and ameliorates the ineffective erythropoiesis seen in $\mathrm{Hbb}^{\text {th } 3 /+}$ mice. The mitapivat-induced improvement in erythropoiesis modulates the ERFE-Hamp axis, resulting in reduction of liver iron overload. In duodenums, mitapivat increases pyruvate kinase activity with reduced expression of PKM2, redirecting cells to a "nonhypoxic" metabolic condition. This reduces the expression of HIF2 $\alpha$ and the activation of NF- $\mathrm{KB}$ p65, both targeting Dmt1 gene expression. Thus, mitapivat treatment blocks both iron absorption and iron release from duodenum, contributing to the reduction of iron overload in $\mathrm{Hbb}^{\mathrm{th} 3 /+}$ mice.

$(P<0.05$ for each; Supplemental Figure 8D). Regarding erythropoiesis in healthy controls, mitapivat did not produce changes in cell numbers (Supplemental Figure 8A), but we observed a trend in accelerating erythroid maturation (Supplemental Figure 8C). At 11 days of cell culture, a reduction in the amount of annexin $\mathrm{V}^{+}$intermediate erythroblasts was observed in healthy control erythroblasts exposed to mitapivat (Supplemental Figure 8D).

These data indicate that mitapivat enhances human $\beta$-thalassemia erythropoiesis, promotes erythroid maturation, and reduces apoptosis.

\section{Discussion}

Metabolic homeostasis is extremely important in a highly dynamic cellular maturation process such as erythropoiesis (49). In $\beta$-thalassemic mouse erythroid cells, we found increased expression of PKM2, which may be part of the compensatory changes induced by the severe oxidative stress $(50,51)$. We reasoned that the pyruvate kinase activator mitapivat may provide additional metabolic resources in terms of more efficient glycolysis, ameliorating $\beta$-thalassemic ineffective erythropoiesis.

Mitapivat treatment ameliorated anemia in $\mathrm{Hbb}^{\mathrm{th} /+}$ mice, increased $\mathrm{Hb}$, and enhanced both red cell survival and ineffective erythropoiesis. An improved morphologic appearance of erythrocytes was accompanied by increased MCV and $\mathrm{MCH}$, linked to the reduction in $\alpha$-globin membrane precipitates and an increase in the fraction of soluble $\mathrm{Hb}$ in red cells from mitapivat-treated $\mathrm{Hbb}^{\mathrm{th} 3 /+}$ mice compared with vehicle-treated animals. This was associated with a reduction in markers of hemolysis and a significant decrease in reticulocyte count $(P<0.05)$. Collectively, these data indicate that mitapivat improves quality control processes during $\beta$-thalassemic erythropoiesis. Indeed, mitapivat treatment of $\mathrm{Hbb}^{\mathrm{th} 3 /+}$ mice produced a reduction in circulating erythroblasts, a marked decrease in extramedullary erythropoiesis, and an improved erythroid maturation index. In both erythropoietic sites (bone marrow and spleen), erythroblast ROS content and apoptosis markers decreased with mitapivat treatment. This was associated with increased ATP in splenic erythroblasts, with a positive trend also in bone marrow erythroblasts from mitapivat-treated $\mathrm{Hbb}^{\mathrm{th} 3 / \mathrm{H}}$ mice.

Of note, mitapivat appeared to beneficially affect mitochondrial dynamics during erythropoiesis in $\beta$-thalassemia. We observed an upregulation of specific mitochondrial genes such as Pgcla, which are known to counteract mitochondrial dysregulation and stimulate mitochondrial biogenesis in erythroblasts (52-54). We also observed downregulation of the expression of Yme1l, a mitochondrial-specific chaperone involved in mitochondrial protein quality control processes $(36,37)$. Taken together, these data indicated that antioxidant compounds may limit ROS cytotoxicity in $\beta$-thalassemia $(1-3,5)$. Indeed, mitapivat appeared to improve metabolic plasticity during erythroid maturation in $\mathrm{Hbb}^{\mathrm{th} 3 /+}$ mice by ensuring cell survival and supporting cell growth. 
The improved erythropoiesis in mitapivat-treated $\mathrm{Hbb}^{\text {th } 3 /+}$ mice is accompanied by substantial changes in iron homeostasis. Perhaps as a consequence of increased responsiveness to EPO and improvements in $\beta$-thalassemia-associated ineffective erythropoiesis, mitapivat treatment resulted in reduced ERFE levels and upregulated Hamp gene expression in the liver. Since we observed upregulation of $I d 1$, we hypothesize that increased Hamp is a consequence of ameliorated ineffective erythropoiesis rather than changes in liver iron content. Consistently, duodenal enterocytes from mitapivat-treated $\mathrm{Hbb}^{\text {th } 3 /+}$ mice exhibited decreased FPN1 content. Interestingly, mitapivat treatment induced a significant reduction in iron duodenum content $(P$ $<0.05)$, combined with decreased expression of PKM2. PKM2 has been shown to translocate into the nucleus as a coactivator of transcription factors such as NF-kB p65 or HIFs $(14,45,51$, 55-57). In duodenums from mitapivat-treated $\mathrm{Hbb}^{\text {th } 3 /+}$ mice, we observed a reduction of both HIF $2 \alpha$ expression and activation of NF-kB p65. These changes were associated with downregulation of Dmt1 expression in duodenums. These data indicate multiple potential targets of pyruvate kinase activation. Mitapivat treatment affects (a) iron absorption through the reduction of both NF-кB p65 and HIF2 $\alpha$ function (58-61), and associated decreased Dmt1 expression; and (b) iron release, by reducing FPN1 protein via hepcidin. Mitapivat should be further investigated as a Dmt1 blocker to control iron absorption in disorders other than $\beta$-thalassemia, such as hemochromatosis (58-60). In addition, as a target of HIF $2 \alpha$, downregulation of Dmt1 and subsequent improvement of anemia may occur via the hepcidin-HIF $2 \alpha$ axis, separately to the involvement of PKM2 (61). Greater clarity surrounding these complex, overlapping mechanisms could be achieved by evaluating the direct effect of mitapivat treatment on PKM2 in WT mice.

In conclusion, we show that the pyruvate kinase activator mitapivat ameliorates $\beta$-thalassemic ineffective erythropoiesis and improves anemia, hemolysis, and red cell survival in $\mathrm{Hbb}^{\text {th } 3 /+}$ mice. Mitapivat improves $\beta$-thalassemic pathologic erythropoiesis and modulates the ERFE-Hamp axis, with reduction of liver iron overload. In the duodenum, mitapivat reduces the expression of PKM2, redirecting cells toward a nonhypoxic metabolic condition with reduced HIF $2 \alpha$ expression and NF- $\mathrm{KB}$ p65 activation, both targeting $D m t 1$ gene expression. Thus, mitapivat treatment positively affects both $\beta$-thalassemic ineffective erythropoiesis and iron homeostasis (Figure 6). It is worth noting that improvement of ineffective erythropoiesis and reduction of spleen erythropoiesis were reported following treatment with either the activin ligand trap RAP-011 or the glycine transporter inhibitor bitopertin (62) in the same $\beta$-thalassemia mouse model used here.

Our in vitro data with $\mathrm{CD}_{3} 4^{+}$erythroid precursors from patients with $\beta$-thalassemia show a similar reduction in ineffective erythropoiesis markers to those observed in the $\mathrm{Hbb}^{\text {th } 3 /+}$ mouse model, further supporting the applicability of our experimental mouse data to the human disease. Interim results of a phase 2 clinical study with orally administered mitapivat (NCT03692052) have recently established proof of concept that pyruvate kinase activation by mitapivat has potential clinical benefit in patients with non-transfusion-dependent thalassemia (29), further confirming the relevance of the present mouse studies.

\section{Methods}

Mouse strains and study design. Two-month-old female WT C57B6 and $\mathrm{Hbb}^{\text {th } 3 /+}$ mice were studied (30). Blood was collected by retro-orbital venipuncture in anesthetized mice using heparinized microcapillary tubes. Hematological parameters, red cell indices, and reticulocyte count were evaluated at baseline and at different time points, as previously reported, on a Siemens ADVIA 2120 Hematology Analyzer. Hematocrit and $\mathrm{Hb}$ were manually determined (31, 48, 63, 64), and $\alpha$-globin membrane aggregates and soluble $\mathrm{Hb}$ were assayed as described by Dussiot et al. (62). The amount of hemichromes bound to the red cell membrane was assessed as previously reported $(31,65)$. Total bilirubin, indirect bilirubin, lactate dehydrogenase, blood urea nitrogen (BUN), and creatinine were evaluated using standard biochemical assays, as previously reported $(5,17,42)$.

To measure survival, red cells from WT or $\mathrm{Hbb}^{\text {th } 3 /+}$ mice treated with vehicle or mitapivat were labeled with CFSE $(10 \mu \mathrm{M}$; Molecular Probes, Invitrogen) in PBS, $0.5 \%$ BSA for 20 minutes at $37^{\circ} \mathrm{C}$, as previously reported (30).

Analysis of reduced GSH and of GSSG in red cells was performed according to the spectrophotometric glutathione reductase recycling procedure previously reported $(66,67)$, with some modifications.

The concentration of mouse EPO in plasma was determined using the Mouse Erythropoietin Quantikine ELISA Kit (R\&D Systems) following the manufacturer instructions. Soluble serum ERFE was determined by ELISA, as detailed in the Supplemental Methods.

Erythrocyte morphology was assessed using May-GrünwaldGiemsa-stained smears that were imaged under oil at $\times 100$ magnification using a PanFluor objective with 1.30 numeric aperture on a Nikon Eclipse DS-5M camera and processed with Nikon Digital Slide (DS-L1).

Measurement of pyruvate kinase activity and ATP. ATP concentration was determined using liquid chromatography tandem mass spectrometry (LC-MS/MS). A 4- $\mu \mathrm{L}$ aliquot of whole blood was mixed with $96 \mu \mathrm{L}$ Milli-Q water. A 10- $\mu \mathrm{L}$ aliquot of the diluted blood was then mixed with $10 \mu \mathrm{L} \mathrm{H}_{2} \mathrm{O}$ and $200 \mu \mathrm{L}$ acetonitrile/ $\mathrm{H}_{2} \mathrm{O}$ (90:10, vol/vol $\left.+0.1 \% \mathrm{NH}_{4} \mathrm{OH}\right)$ containing the internal standards ${ }^{13} \mathrm{C}_{3}$-2,3-diphosphoglycerate $(5 \mu \mathrm{g} / \mathrm{mL})$ and ${ }^{13} \mathrm{C}_{10},{ }^{15} \mathrm{~N}_{5}$-ATP $(2.5 \mu \mathrm{g} / \mathrm{mL})$. The mixture was vortexed at $1500 \mathrm{rpm}$ for 2 minutes and centrifuged at $5228 \mathrm{~g}$ for 5 minutes. A 7.5- $\mu \mathrm{L}$ aliquot of supernatant was injected for analysis by LC-MS/MS. A reversed-phase gradient method was used to provide sample stacking and separation. Calibration curves were established using ATP standards. The peak area ratios of the analyte relative to the internal standard were used for quantitation.

Pyruvate kinase activity measurements were performed as described (28), except $50 \mu \mathrm{M}$ of phosphoenolpyruvate was used.

Flow cytometric analysis of mouse erythroid precursors and molecular analysis of sorted erythroid cells. Flow cytometric analysis of erythroid precursors from bone marrow and spleen from WT and $\mathrm{Hbb}^{\text {th } 3 /+}$ mice was carried out using the CD44-Ter119 schema $(17,68,69)$. This strategy allows identification of 4 different erythroblast subpopulations: population I, II, III, and IV, respectively corresponding to proerythroblasts, basophilic, polychromatic, and orthochromatic erythroblasts. Analysis of apoptotic orthochromatic erythroblasts was carried out on the CD44-Ter119 gated cells using the Annexin-V PE Apoptosis detection kit (eBioscience) following the manufacturer's instructions. Erythroblast ROS levels were evaluated as previously reported using the general oxidative stress indicator, CM-H2DCFDA $(10 \mu \mathrm{M}$, Thermo Fisher Scientific) on CD44-Ter119 gated populations (17). Flow 
cytometric analysis of the mitochondria was carried out using the MitoTracker Deep Red probe (Thermo Fisher Scientific), following the manufacturer instructions. Details of the cytometric analysis and subsequent Western blot, quantitative real-time PCR (qRT-PCR), and mitochondrial analyses are given in the Supplemental Methods.

Molecular analysis of liver and C-duodenum. Protocols used for RNA isolation, cDNA preparation, and qRT-PCR have been previously described $(30,48,62)$. qRT-PCR was performed using the SYBR-green method as detailed in the Supplemental Methods, with all primer sequences shown in Supplemental Table 3. Western blots were performed as described in the Supplemental Methods.

Immediately following dissection, the liver was formalin-fixed and paraffin-embedded for Perl's staining (17). Details of quantification of spleen and liver iron content and the ferroportin (Fpn1) immunohistochemistry assay are given in the Supplemental Methods.

In vitro human erythropoiesis. We analyzed 10 erythroid cultures obtained from the peripheral blood of different healthy subjects and 10 erythroid cultures obtained from 4 homozygous $\beta$-thalassemia intermedia patients $\left(\beta^{\text {ocod39}}\right)$ who were referred to our center $(48)$. The erythroid cell antigen profiling, the sorting of erythroid precursors, and cell culture were carried out as previously described (48) and as detailed in the Supplemental Methods. We then carried out preliminary studies of various concentrations, timings, and frequencies of addition of mitapivat for in vitro human $\beta$-thalassemia erythropoiesis testing. Since the number of $\mathrm{CD} 34^{+}$cells derivable from peripheral blood of $\beta$-thalassemia intermedia patients was too low, the doseresponse experiments were carried out only in cells from healthy subjects. The preliminary experiments resulted in a protocol adding mitapivat at days $5,7,10$, and 13 of the cell cultures.

Statistics. Data were analyzed using either $t$ test or 1-way ANOVA (Dunnett's test) for longitudinal studies or 2-way ANOVA with Bonferroni connection for repeated measures between the mice of various genotypes, as well as between healthy and $\beta$-thalassemic cells. A difference with a $P$ less than 0.05 was considered significant.

Study approval. The Institutional Animal Experimental Committee of University of Verona (CIRSAL) and the Italian Ministry of Health approved the experimental protocols (56DC9.12), following European directive 2010/63/EU and the Federation for Laboratory Animal Science Associations guidelines and recommendations. The study was approved by the Ethical Committee of the Azienda Ospedaliera Integrata Verona, Italy (FGRF13IT). Written informed consent was obtained from patients with $\beta$-thalassemia and healthy controls.

\section{Author contributions}

GF, FC, MAD, LS, CL, MTV, AJ, AI, CB, and LDF wrote the manuscript. AM, CB, and LDF designed the experiments. SY and SR carried out 2,3-DPG and ATP determinations. AM carried out standard hematology, cytofluorimetric analysis, ELISA, and Western blot experiments. EF and II carried out immunoblot experiments. PAK carried out pyruvate kinase activity experiments and contributed to manuscript revision. RN carried out ATP experiments. $\mathrm{CK}$ and LD contributed to therapeutic concept and manuscript revision. TG and CLJ carried out soluble ERFE measurements and contributed to manuscript revision. RR and AI carried out RT-PCR analyses. GF and FC carried out Fpn1 studies. MAD and LS carried out mitochondria studies. FT carried out the analyses of hemichromes. AJ and CL carried out blinded pathologic analyses. EB carried out CD34-derived liquid cultures. AM, EF, CK, PAK, GF, FC, MAD, LS, MTV, FT, EB, II, LD, TG, CLJ, AI, CB, and LDF analyzed data.

\section{Acknowledgments}

This study was supported by an Agios Pharmaceuticals, Inc. research collaborative grant to LDF. Editorial assistance was provided by Christine Ingleby, Excel Medical Affairs, Horsham, United Kingdom, who was supported by Agios.

Address correspondence to: Carlo Brugnara, Department of Laboratory Medicine, Boston Children's Hospital, Harvard Medical School, 300 Longwood Avenue, Boston, Massachusetts 02115, USA. Phone: 1.617.355.6610; Email: carlo.brugnara@childrens. harvard.edu.
1. Rund D, Rachmilewitz E. $\beta$-thalassemia. N Engl J Med. 2005;353(11):1135-1146.

2. Rivella S. $\beta$-thalassemias: paradigmatic diseases for scientific discoveries and development of innovative therapies. Haematologica. 2015;100(4):418-430.

3. De Franceschi L, et al. Oxidative stress and $\beta$-thalassemic erythroid cells behind the molecular defect. Oxid Med Cell Longev. 2013;2013:985210.

4. Zhang X, et al. FOXO3-mTOR metabolic cooperation in the regulation of erythroid cell maturation and homeostasis. Am J Hematol. 2014;89(10):954-963.

5. Matte A, et al. Bitopertin, a selective oral GLYT1 inhibitor, improves anemia in a mouse model of $\beta$-thalassemia. JCI Insight. 2019;4(22):e130111.

6. Kim HD, et al. Metabolic adaptation during erythropoietin-mediated terminal differentiation of mouse erythroid cells. Blood. 1991;77(2):387-392.

7. Brown JE, Adamson JW. Modulation of in vitro erythropoiesis: enhancement of erythroid colony growth by cyclic nucleotides. Cell Tissue Kinet.
1977;10(3):289-298.

8. Brown JE, Adamson JW. Studies of the influence of cyclic nucleotides on in vitro haemoglobin synthesis. Br J Haematol. 1977;35(2):193-208.

9. Nijhof W, et al. Changes in activities and isozyme patterns of glycolytic enzymes during erythroid differentiation in vitro. Blood. 1984;64(3):607-613.

10. Ting YL, et al. In vivo metabolic studies of glucose, ATP and 2,3-DPG in beta-thalassaemia intermedia, heterozygous beta-thalassaemic and normal erythrocytes: 13C and 31P MRS studies. Br J Haematol. 1994;88(3):547-554.

11. Chakraborty I, et al. Distortion of $\beta$-globin chain of hemoglobin alters the pathway of erythrocytic glucose metabolism through band 3 protein. Arch Med Res. 2012;43(2):112-116.

12. Khungwanmaythawee $\mathrm{K}$, et al. Mitochondrial changes in $\beta 0$-Thalassemia/Hb E disease. PLoS One. 2016;11(4):e0153831.

13. Anastasiou D, et al. Inhibition of pyruvate kinase M2 by reactive oxygen species contributes to cellular antioxidant responses. Science. 2011;334(6060):1278-1283.
14. Christofk HR, et al. The M2 splice isoform of pyruvate kinase is important for cancer metabolism and tumour growth. Nature. 2008;452(7184):230-233.

15. Chakraborty D, Bhattacharyya M. Antioxidant defense status of red blood cells of patients with beta-thalassemia and Ebeta-thalassemia. Clin Chim Acta. 2001;305(1-2):123-129.

16. Ponnazhagan S, Sarkar R. Enzymes of the pentose phosphate pathway in glutathione-regulated membrane protection in beta-thalassaemia. Eur JClin Chem Clin Biochem. 1992;30(8):481-484.

17. Matte A, et al. Peroxiredoxin-2: a novel regulator of iron homeostasis in ineffective erythropoiesis. Antioxid Redox Signal. 2018;28(1):1-14.

18. Yang W, Lu Z. Pyruvate kinase M2 at a glance. JCell Sci. 2015;128(9):1655-1660.

19. Zhang Z, et al. PKM2, function and expression and regulation. Cell Biosci. 2019;9:52.

20. Ogier $\mathrm{H}$, et al. Dietary and hormonal regulation of L-type pyruvate kinase gene expression in rat small intestine. Eur J Biochem. 1987;166(2):365-370.

21. Klei TRL, et al. Residual pyruvate kinase activity 
in PKLR-deficient erythroid precursors of a patient suffering from severe haemolytic anaemia. Eur J Haematol. 2017;98(6):584-589.

22. Aizawa $S$, et al. Ineffective erythropoiesis in the spleen of a patient with pyruvate kinase deficiency. Am J Hematol. 2003;74(1):68-72.

23. Aizawa $\mathrm{S}$, et al. Ineffective erythropoiesis in mutant mice with deficient pyruvate kinase activity. Exp Hematol. 2005;33(11):1292-1298.

24. Max-Audit I, et al. Pyruvate kinase synthesis and degradation by normal and pathologic cells during erythroid maturation. Blood. 1988;72(3):1039-1044.

25. Tsujino K, et al. Delayed onset of hemolytic anemia in CBA-Pk-1slc/Pk-1slc mice with a point mutation of the gene encoding red blood cell type pyruvate kinase. Blood.1998;91(6):2169-2174.

26. Grace RF, et al. Safety and efficacy of mitapivat in pyruvate kinase deficiency. $N$ Engl J Med. 2019;381(10):933-944.

27. Yang $\mathrm{H}$, et al. Phase 1 single- and multipleascending-dose randomized studies of the safety, pharmacokinetics, and pharmacodynamics of AG-348, a first-in-class allosteric activator of pyruvate kinase $\mathrm{R}$, in healthy volunteers. Clin Pharmacol Drug Dev. 2019;8(2):246-259.

28. Kung C, et al. AG-348 enhances pyruvate kinase activity in red blood cells from patients with pyruvate kinase deficiency. Blood. 2017;130(11):1347-1356.

29. Kuo KHM, et al. Proof of concept for the oral pyruvate kinase activator mitapivat in adults with non-transfusion-dependent thalassemia: Interim results from an ongoing, phase 2, open-label, multicenter study. Paper presented at: 62nd American Society of Hematology Annual Meeting and Exposition; December 5-8, 2020; Virtual. https://www.agios.com/wp-content/ uploads/2020/12/Abstract-2600_Proof-ofConcept-for-the-Oral-PK-Activator-Mitapivatin-Thalassemia.pdf. Accessed January 15, 2021.

30. Matte A, et al. The interplay between peroxiredoxin-2 and nuclear factor-erythroid 2 is important in limiting oxidative mediated dysfunction in $\beta$-thalassemic erythropoiesis. Antioxid Redox Signal. 2015;23(16):1284-1297.

31. Matte A, et al. Peroxiredoxin-2 expression is increased in beta-thalassemic mouse red cells but is displaced from the membrane as a marker of oxidative stress. Free Radic Biol Med. 2010;49(3):457-466.

32. Olivieri O, et al. Oxidative damage and erythrocyte membrane transport abnormalities in thalassemias. Blood. 1994;84(1):315-320.

33. Iqbal MA, et al. Insulin enhances metabolic capacities of cancer cells by dual regulation of glycolytic enzyme pyruvate kinase M2. Mol Cancer. 2013;12:72.

34. Wang YH, et al. Cell-state-specific metabolic dependency in hematopoiesis and leukemogenesis. Cell. 2014;158(6):1309-1323.

35. La P, et al. An orchestrated balance between mitochondria biogenesis, iron-sulfur cluster synthesis and cellular iron acquisition. Blood. 2018;132(suppl 1):1048.

36. Ruan Y, et al. Loss of Yme1L perturbates mitochondrial dynamics. Cell Death Dis. 2013;4(10):e896.

37. Stiburek L, et al. YME1L controls the accumulation of respiratory chain subunits and is required for apoptotic resistance, cristae morphogenesis, and cell proliferation. Mol Biol Cell. 2012;23(6):1010-1023.

38. Casu C, et al. Minihepcidin peptides as disease modifiers in mice affected by $\beta$-thalassemia and polycythemia vera. Blood. 2016;128(2):265-276.

39. Nai A, et al. Limiting hepatic Bmp-Smad signaling by matriptase- 2 is required for erythropoietin-mediated hepcidin suppression in mice. Blood. 2016;127(19):2327-2336.

40. Aschemeyer S, et al. Erythroferrone and matriptase- 2 independently regulate hepcidin expression. Am J Hematol. 2017;92(5):E61-E63.

41. Gardenghi S, et al. Ineffective erythropoiesis in beta-thalassemia is characterized by increased iron absorption mediated by down-regulation of hepcidin and up-regulation of ferroportin. Blood. 2007;109(11):5027-5035.

42. Bellelli R, et al. NCOA4 deficiency impairs systemic iron homeostasis. Cell Rep. 2016;14(3):411-421.

43. Drakesmith $\mathrm{H}$, et al. Ironing out ferroportin. Cell Metab. 2015;22(5):777-787.

44. Palsson-McDermott EM, et al. Pyruvate kinase M2 regulates Hif-1alpha activity and IL-1beta induction and is a critical determinant of the warburg effect in LPS-activated macrophages. Cell Metab. 2015;21(1):65-80.

45. Luo W, et al. Pyruvate kinase M2 is a PHD3-stimulated coactivator for hypoxia-inducible factor 1 Cell. 2011;145(5):732-744.

46. De Franceschi L, et al. K-CL co-transport plays an important role in normal and beta thalassemic erythropoiesis. Haematologica. 2007;92(10):1319-1326.

47. De Franceschi L, et al. Oxidative stress modulates heme synthesis and induces peroxiredoxin-2 as a novel cytoprotective response in B-thalassemic erythropoiesis. Haematologica. 2011;96(11):1595-1604.

48. Franco SS, et al. Resveratrol accelerates erythroid maturation by activation of FoxO3 and ameliorates anemia in beta-thalassemic mice. Haematologica. 2014;99(2):267-275.

49. Liu X, et al. Regulation of mitochondrial biogenesis in erythropoiesis by mTORC1-mediated protein translation. Nat Cell Biol. 2017;19(6):626-638.

50. Dong G, et al. PKM2 and cancer: the function of PKM2 beyond glycolysis. Oncol Lett. 2016;11(3):1980-1986.

51. Azoitei $\mathrm{N}$, et al. PKM2 promotes tumor angiogenesis by regulating HIF- $1 \alpha$ through NF-KB activation. Mol Cancer. 2016;15:3.

52. Yang JL, et al. Diverse roles of mitochondria in ischemic stroke. Redox Biol. 2018;16:263-275.

53. Whitaker RM, et al. Mitochondrial biogenesis as a pharmacological target: a new approach to acute and chronic diseases. Annu Rev Pharmacol Toxicol. 2016;56:229-249.

54. Cameron RB, et al. Development of therapeutics that induce mitochondrial biogenesis for the treatment of acute and chronic degenerative diseases. J Med Chem. 2016;59(23):10411-10434.

55 . Wang D, et al. Hypoxia regulates the ferrous iron uptake and reactive oxygen species level via divalent metal transporter 1 (DMT1) Exon1B by hypoxia-inducible factor-1. IUBMB Life. 2010;62(8):629-636

56. Dengler VL, et al. Transcriptional regulation by hypoxia inducible factors. Crit Rev Biochem Mol Biol. 2014;49(1):1-15.

57. Xu Q, et al. Regulatory circuit of PKM2/NF-kB/ miR-148a/152-modulated tumor angiogenesis and cancer progression. Oncogene. 2015;34(43):5482-5493.

58. Paradkar PN, Roth JA. Nitric oxide transcriptionally down-regulates specific isoforms of divalen metal transporter (DMT1) via NF-kappaB. J Neurochem. 2006;96(6):1768-1777.

59. Anderson ER, et al. Intestinal HIF2 $\alpha$ promotes tissue-iron accumulation in disorders of iron overload with anemia. Proc Natl Acad Sci U S A. 2013;110(50):E4922-E4930.

60. Kumfu S, et al. Effects of iron overload condition on liver toxicity and hepcidin/ferroportin expression in thalassemic mice. Life Sci. 2016;150:15-23.

61. Schwartz AJ, et al. Hepatic hepcidin/intestinal HIF- $2 \alpha$ axis maintains iron absorption during iron deficiency and overload. J Clin Invest. 2019;129(1):336-348.

62. Dussiot $\mathrm{M}$, et al. An activin receptor IIA ligand trap corrects ineffective erythropoiesis in $\beta$-thalassemia. Nat Med.2014;20(4):398-407.

63. de Franceschi $L$, et al. In vivo reduction of erythrocyte oxidant stress in a murine model of beta-thalassemia. Haematologica. 2004;89(11):1287-1298.

64. Dalle Carbonare L, et al. Hypoxia-reperfusion affects osteogenic lineage and promotes sickle cell bone disease. Blood. 2015;126(20):2320-2328.

65. Matté A, et al. The novel role of peroxiredoxin-2 in red cell membrane protein homeostasis and senescence. Free Radic Biol Med. 2014;76:80-88

66. Giustarini D, et al. Analysis of GSH and GSSG after derivatization with $\mathrm{N}$-ethylmaleimide. Nat Protoc. 2013;8(9):1660-1669.

67. Shaik IH, Mehvar R. Rapid determination of reduced and oxidized glutathione levels using a new thiol-masking reagent and the enzymatic recycling method: application to the rat liver and bile samples. Anal Bioanal Chem. 2006;385(1):105-113

68. Liu J, et al. Quantitative analysis of murine terminal erythroid differentiation in vivo: novel method to study normal and disordered erythropoiesis. Blood.2013;121(8):e43-e49.

69. Konstantinidis DG, et al. Cytokinesis failure in RhoA-deficient mouse erythroblasts involves actomyosin and midbody dysregulation and triggers p53 activation. Blood.2015;126(12):1473-1482. 